\title{
Microchannel Heat Transfer with Slip Flow and Wall Effects
}

\author{
Kevin D. Cole* \\ University of Nebraska-Lincoln, Lincoln, Nebraska 68588-0526 \\ Barbaros Çetin $\ddagger$ \\ Bilkent University, Ankara 06800, Turkey \\ and \\ Lukas Brettmann $\stackrel{\ddagger}{\sharp}$ \\ University of Nebraska-Lincoln, Lincoln, Nebraska 68588-0526 \\ DOI: $10.2514 / 1 . T 4267$
}

\begin{abstract}
Analysis is presented for conjugate heat transfer in a parallel-plate microchannel. Axial conduction in the fluid and in the adjacent wall is included. The fluid is a constant property gas with a slip-flow velocity distribution. The microchannel is heated by a small region on the channel wall. The analytic solution is given in the form of integrals by the method of Green's functions. Quadrature is used to obtain numerical results for the temperature and heat transfer coefficient on the heated region for various Peclet number, Knudsen number, and wall materials. A region downstream of the heater is also explored. These results have application in the optimal design of small-scale heat transfer devices for biomedical applications, electronic cooling, and advanced fuel cells.
\end{abstract}

\section{Nomenclature}

\begin{tabular}{|c|c|c|}
\hline 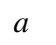 & $=$ & size of heated region along the channel, $\mathrm{m}$ \\
\hline$B_{0}$ & $=$ & effect of internal heating \\
\hline$G$ & $=$ & steady Green's function \\
\hline$g$ & $=$ & volume energy generation, $\mathrm{W} \mathrm{m}^{-3}$ \\
\hline$h$ & $=$ & heat transfer coefficient, $\mathrm{W} /\left(\mathrm{m}^{2} \mathrm{~K}\right)$ \\
\hline$j$ & $=$ & imaginary number, $\sqrt{-1}$ \\
\hline$J$ & $=$ & thermal conductivity, $\mathrm{W} \mathrm{m}^{-1} \mathrm{~K}^{-1}$ \\
\hline$K n$ & $=$ & Knudsen number, $\lambda / L$ \\
\hline$L$ & $=$ & channel height, $\mathrm{m}$ \\
\hline$L_{i}$ & $=$ & thickness of layer $i, \mathrm{~m}$ \\
\hline$N$ & $=$ & number of layers in fluid flow \\
\hline$P e$ & $=$ & Peclet number, $U L / \alpha_{1}$ \\
\hline$p_{0}$ & $=$ & introduced heat flux, $\mathrm{W} \mathrm{m}^{-2}$ \\
\hline$q_{i j}$ & $=$ & heat flux, layer $i$ to $j, \mathrm{~W} \mathrm{~m}^{-2}$ \\
\hline$r$ & $=$ & contact parameter \\
\hline$R_{0}$ & $=$ & contact resistance, $\mathrm{m}^{2} \mathrm{~K} \mathrm{~W}^{-1}$ \\
\hline$T$ & $=$ & temperature, $\mathrm{K}$ \\
\hline$U$ & $=$ & average velocity, $\mathrm{m} / \mathrm{s}$ \\
\hline$u$ & $=$ & local velocity, $\mathrm{m} / \mathrm{s}$ \\
\hline$w$ & $=$ & wall thickness, also $L_{0}, \mathrm{~m}$ \\
\hline$\alpha$ & $=$ & thermal diffusivity, $\mathrm{m}^{2} \mathrm{~s}^{-1}$ \\
\hline$\beta$ & $=$ & wave number, Eq. (ㅇ), $\mathrm{m}^{-1}$ \\
\hline$\delta$ & $=$ & Dirac delta function \\
\hline$\lambda$ & $=$ & mean free path, $\mathrm{m}$ \\
\hline$\omega$ & $=$ & frequency, $\mathrm{s}^{-1}$ \\
\hline
\end{tabular}

Superscripts

$+\quad=$ dimensionless quantity

$-\quad=\quad$ spatial Fourier transform, Eq. (9)

Received 29 August 2013; revision received 24 December 2013; accepted for publication 1 February 2014; published online 10 April 2014. Copyright (C) 2013 by Kevin D. Cole. Published by the American Institute of Aeronautics and Astronautics, Inc., with permission. Copies of this paper may be made for personal or internal use, on condition that the copier pay the $\$ 10.00$ per-copy fee to the Copyright Clearance Center, Inc., 222 Rosewood Drive, Danvers, MA 01923; include the code 1533-6808/14 and \$10.00 in correspondence with the CCC.

*Mechanical and Materials Engineering Department, W342 Nebraska Hall; kcole1@unl.edu (Corresponding Author).

${ }^{\dagger}$ Mechanical Engineering Department, Microfluidics and Lab-on-a-Chip Research Group.

${ }^{\ddagger}$ Mechanical and Materials Engineering Department.

Subscripts
$\begin{array}{lll}\text { av }= & \text { average } \\ i & = & \text { within layer } i \\ 0 & = & \text { wall value } \\ 1 & = & \text { fluid value }\end{array}$

\section{Introduction}

A S FLUID flow and heat transfer take place at the microscale, many additional effects such as rarefaction, electroviscous effects, viscous dissipation, axial conduction, etc. need to be considered, which can be neglected at the macroscale.

From the heat transport point of view, the characteristic time for convection and conduction become comparable at the microscale, and the convection term no longer dominates the conduction term in the longitudinal direction. This is defined by flow for which the Peclet number is not too large. Under this condition, axial conduction in the fluid cannot be neglected, as in the case of macrochannel flow. The effect of the axial conduction in the fluid becomes more pronounced as $P e$ decreases. The effect of axial conduction in the fluid on the heat transfer has been studied for both parallel-plate microchannel [1] and microtube $[2,3]$ for boundary conditions defined by constant wall temperature $[1,2]$ and constant wall heat flux $[1,3]$.

In conventional applications involving channels, the channel wall thickness is very small compared to the hydraulic diameter of the channel; hence, the heat transferred by conduction in the wall can be neglected compared to the convective heat transfer in many macroscale flows. However, in microchannels, the thickness of the channel wall is never negligible compared to the channel size because of rigidity and fabrication concerns. Therefore, the heat transferred in the wall by conduction cannot be neglected, especially for gas flows. The effect of axial conduction in the wall has been studied for macrochannel flows [4,5]. In these studies, corresponding $P e$ values are high and, as a consequence, the axial conduction in the fluid was neglected. Maranzana et al. [6], Kroeker et al. [7] , Li et al. [8], Kim and $\operatorname{Kim}[9,10]$ studied the effect of axial conduction at the wall for the microchannel heat sinks for both circular [7] and rectangular $[\underline{6}, \underline{8}, \underline{9}, 10]$ channel geometries. Maranzana et al. [6] studied the influence of axial conduction for parallel-plate geometry. Some of these studies $[\underline{6}, \underline{9}, 10]$ had an assumption of constant convective heat transfer coefficient at the channel wall, that is, the linkage between the channel wall and the fluid flow was treated approximately. In contrast, Nonino et al. [11] analyzed the circular microtube using conjugate heat transfer, for which no approximation was introduced 
at the fluid-wall boundary. Recently, Kosar [12] analyzed the effect of the wall thickness and the wall material on heat transfer mechanism for a rectangular geometry with a fixed size, that is, only one geometry was studied. Although the results are presented in terms of nondimensional quantities, the analysis was dimensional. Moreover, the thermal boundary condition at the exit of the microchannel was specified convective flux, which was appropriate for the high $\mathrm{Pe}$ range and high $R e$ range $(100<R e<1800)$ to which the work was restricted.

Rarefaction is important for small dimensions compared to the mean free path of the fluid (less than $5 \mu \mathrm{m}$ at atmospheric conditions), and is common for gas flows in microchannels. Electroviscous effects are due to the interaction of the ions in the fluid with the electrical double layer near the nonconducting channel wall [13], and are significant for liquid flow in microchannels with dimensions less than $5 \mu \mathrm{m}$ for deionized water. Viscous dissipation is the heating of the fluid due to the work done against the viscous forces. The effect of viscous dissipation can be important for flows with Reynolds number greater than 100 for microchannels [14]. A recent review paper by Colin [15] is focused specifically on gas microflows for which slip flow is present. Colin identifies several areas of active research in the slip-flow regime, including viscous dissipation, entrance effects, and the effect of shear work at the wall. However, Colin's review is limited to gas flow with specified boundary temperature or specified boundary flux, that is, that heat transfer in the adjacent wall is not discussed. For gas flows, and especially for short heating in the axial direction, the effect of the wall can play a significant role in defining the heat transfer.

In this study, the heat transfer to a gas flow inside a parallel-plate microchannel for low $P e$ number flow is analyzed. The effect of axial conduction in both the gas and the wall is considered. Exact analytical solutions for the temperature distribution in the fluid and the wall are obtained by using the Green's function method. The solution has the form of integrals and quadrature is used to obtain numerical values. Local and average values of the fluid temperature are determined for a range of fluid flow values and for several wall materials. This information is expected to be useful in the analysis and design of microscale heat transfer devices.

The unique contributions of this paper are the following: gas flow in the slip-flow regime is studied over a range of Peclet numbers such that fluid axial conduction is included; wall effects (also called conjugate heat transfer) are included; a short heated region along the wall is investigated and the convection effects downstream are investigated; the analytic solution provides high precision, if desired; and a wide range of results is explored because the quadrature may be evaluated rapidly compared to a fully numeric solution.

\section{Temperature Equations}

The equations describing the temperature in the parallel-plate flow and in the adjacent wall are given in this section. The geometry is shown in Fig. 1. A heater is embedded in the channel wall heated and the flow between parallel plates is fully developed laminar. The plate spacing is $L$ and the wall thickness is $L_{0}$. The theoretical discussion given next is similar to that developed previously [16], so only a brief outline is given. The discussion is limited to steady-periodic heating and steady periodic temperature. The temperature satisfies the following equations

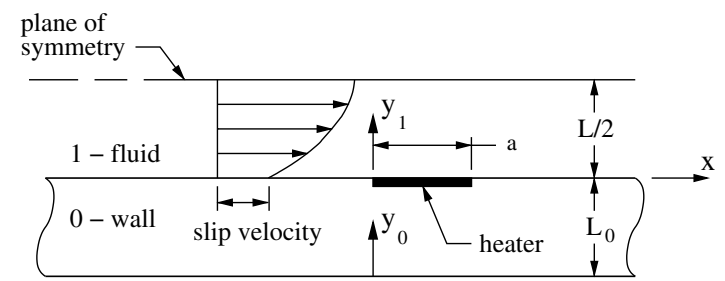

Fig. 1 Geometry of parallel plates and slip flow with heating over a thin layer at fluid-wall interface.

$$
\frac{\partial^{2} T_{0}}{\partial x^{2}}+\frac{\partial^{2} T_{0}}{\partial y_{0}^{2}}=-\frac{g\left(x, y_{0}, \omega\right)}{k_{0}}+\frac{j \omega}{\alpha_{0}} T_{0} ; \quad 0<y_{0}<L_{0} ; \quad-\infty<x<\infty
$$

$$
\frac{\partial^{2} T_{1}}{\partial x^{2}}+\frac{\partial^{2} T_{1}}{\partial y_{1}^{2}}=\frac{u\left(y_{1}\right)}{\alpha_{1}} \frac{\partial T_{1}}{\partial x}+\frac{j \omega}{\alpha_{1}} T_{1} ; \quad 0<y_{1}<L / 2 ; \quad-\infty<x<\infty
$$

$$
\left.\frac{\partial T_{0}}{\partial y_{0}}\right|_{y_{0}=0}=0 ;\left.\quad \frac{\partial T_{1}}{\partial y_{1}}\right|_{y_{1}=L / 2}=0 \quad T(x \rightarrow \pm \infty) \text { is bounded }
$$

Here, complex-valued temperature $T_{i}(x, y, \omega)$ is interpreted to be the steady-periodic temperature in the $i$ th body at single frequency $\omega$. Later in the paper, results will be discussed as the amplitude of this temperature. The specified volumetric heating $g\left(x, y_{0}, \omega\right)$ represents heating introduced inside the channel wall at frequency $\omega$. The outer surface of the channel wall is insulated, and the centerline of the fluid flow is a zero-flux boundary to represent a channel that is heated symmetrically.

In this paper, the specific case of slip flow is treated. The fully developed velocity distribution for slip flow between parallel plates is given by

$$
\frac{u}{U}=6 \frac{y / L-(y / L)^{2}+12 K n}{1+12 K n}
$$

where $U$ is the mean velocity and $K n=\lambda / L$ is the Knudsen number [17]. The velocity field is shown in Fig. 2. Note that for $K n=0$, the velocity reduces to simple laminar flow and for $K n=0.08$, the velocity jump at the wall is $50 \%$ of the average-flow velocity.

The preceding differential equations for the temperature will next be recast as integral equations in each region (solid, fluid) with the method of Green's functions.

\section{Green's Function Solution}

In this section, Green's function method will be used to seek the temperature distribution in the fluid and the adjacent wall. The Green's function in each region is a solution to the same equations and boundary conditions as those satisfied by the temperature, except that the distributed heat source is replaced by a point heat source. The temperature solution is assembled by adding together many Green's functions in such a way that the heating distribution $g\left(x, y_{0}\right)$ is reconstructed from point sources. This adding together takes the form of a superposition integral, as shown next.

\section{A. Two-Layer Solution}

This solution will be sought by treating the fluid and the wall separately and by treating the heat flux flowing between the fluid and the wall as unknown quantities to be determined from the solution. Let region 0 be a stationary solid heated by a distributed heat source $g\left(x, y_{0}\right)$. Let region 1 be a flowing fluid that is heated by contact with

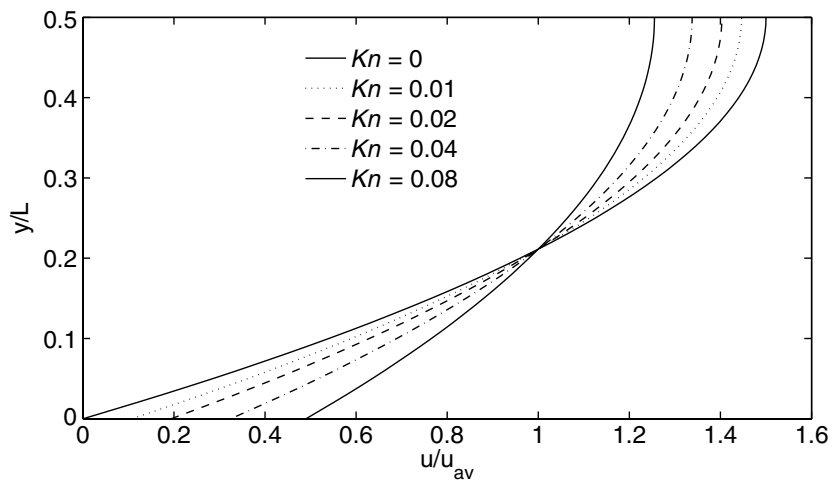

Fig. 2 Velocity distribution showing slip velocity at wall for $K n>0$. 
region 0 . Then the temperatures in each region may be formally stated with the method of Green's functions (GF), in terms of known GF named $G_{1}$ and $G_{0}$ and unknown interface heat fluxes $q_{10}$ and $q_{01}$, as follows [1]

$$
\begin{gathered}
T_{1}\left(x, y_{1}\right)=\frac{1}{k_{1}} \int q_{10}\left(x^{\prime}\right) G_{1}\left(x-x^{\prime}, y_{1}, y_{1}^{\prime}=0\right) \mathrm{d} x^{\prime} \\
T_{0}\left(x, y_{0}\right)=\frac{1}{k_{0}} \int q_{01}\left(x^{\prime}\right) G_{0}\left(x-x^{\prime}, y_{0}, y^{\prime}{ }_{0}=L_{0}\right) \mathrm{d} x^{\prime}+B_{0}\left(x, y_{0}\right)
\end{gathered}
$$

where

$$
B_{0}\left(x, y_{0}\right)=\frac{1}{k_{0}} \iint g\left(x^{\prime}, y_{0}^{\prime}\right) G_{0}\left(x-x^{\prime}, y_{0}, y_{0}^{\prime}\right) \mathrm{d} x^{\prime} \mathrm{d} y^{\prime}
$$

Here, $B_{0}$ is the contribution to the temperature caused by the (known) distributed heat source $g\left(x, y_{0}\right)$. In the preceding equations, the interface heat fluxes and the interface temperatures are unknown, but they are related by matching conditions at the interface between the regions. The heat flux entering region 1 leaves region 0

$$
q_{01}(x)=-q_{10}(x)
$$

There is a temperature jump at the fluid-solid interface in slip flow, given by

$$
\begin{gathered}
T_{0}\left(x, L_{0}\right)-T_{1}(x, 0)=\left.r \lambda \frac{\partial T_{1}}{\partial y_{1}}\right|_{y_{1}=0} \\
\text { where } r=\frac{2-F_{T}}{F_{T}} \frac{2 \gamma}{\gamma+1} \frac{1}{P r}
\end{gathered}
$$

Here, $F_{T}$ is the thermal accommodation factor, $\gamma$ is the specific heat ratio, and $P r$ is the Prandtl number of the fluid [3]. For $K n=0$, the jump in wall temperature vanishes.

Next, the Fourier transform will be used to strip away the integrals. The Fourier transform is defined by the following transform pair

$$
\begin{gathered}
\bar{T}(\beta)=\int_{-\infty}^{\infty} T(x) e^{-j \beta x} \mathrm{~d} x \\
T(x)=\frac{1}{2 \pi} \int_{-\infty}^{\infty} \bar{T}(\beta) e^{j \beta x} \mathrm{~d} \beta
\end{gathered}
$$

Apply the Fourier transform to Eqs. (ㅁ-몽 to obtain

$$
\begin{gathered}
\bar{T}_{1}\left(\beta, y_{1}\right)=\frac{1}{k_{1}} \bar{q}_{10}(\beta) \bar{G}_{1}\left(y_{1}, y^{\prime}{ }_{1}=0\right) \\
\bar{T}_{0}\left(\beta, y_{0}\right)=\frac{1}{k_{0}} \bar{q}_{01}(\beta) \bar{G}_{0}\left(y_{0}, y^{\prime}{ }_{0}=L_{0}\right)+\bar{B}_{0}\left(\beta, y_{0}\right) \\
\bar{q}_{01}(\beta)=-\bar{q}_{10}(\beta) \\
\bar{T}_{0}\left(x, L_{0}\right)-\bar{T}_{1}(x, 0)=\left.r \lambda \frac{\partial \bar{T}_{1}}{\partial y_{1}}\right|_{y_{1}=0}
\end{gathered}
$$

If the GF are known in Fourier space, then an algebraic solution can be obtained for the unknown interface temperatures and heat fluxes. In the next section, the GF for the fluid flow is found from a layered description of the fluid flow.

\section{B. Multiple-Layer Solution}

It is possible to define one GF to describe the temperature in a channel with a continuously varying velocity distribution. This approach requires use of a series involving the hypergeometric function with challenging series convergence behavior [16]. In contrast, the layered approach given here involves a closed-form GF in each layer combined with a simple matrix solution. The smooth velocity distribution in the fluid will be replaced by a collection of flat layers, each one sliding over its neighbors with piecewise constant velocity. In each of these layers, application of the Fourier transform removes the $x$ coordinate, leaving heat conduction through layers along the $y$ direction. This method has been previously applied to laminar flow in liquids [19], and the present work is an extension of the method to slip flow in gases.

The layered geometry shown in Fig. 3 has $N+1$ layers, numbered from 0 to $N$, with $N$ interfaces between the layers. If the zeroth layer is taken to be the wall with zero velocity, then the description of the wall can be included. Layers 1 through $N$ are located in the gas flow, with uniform velocity in each layer set to a value to produce a piecewise version of the slip-flow velocity distribution. Layer $i$ has thickness $L_{i}$ and thermal properties $k_{i}$ and $\alpha_{i}$. Within layer $i$, the interfaces are at local coordinates $y_{i}=0$ and $y_{i}=L_{i}$. At the interfaces between the layers, let $q_{n m}$ represent the heat flux leaving layer $n$ and entering layer $m$. In the formulation given next, heating is introduced in layer 0 (the wall) and the dependence on Fourier parameter $\beta$ is dropped to streamline the development. Although in this formulation there is an insulated condition provided at the top of layer $N$, another stationary wall could easily be added with heating or cooling included at that point.

Consider first the temperature in layer 0 evaluated at its interface with layer 1

$$
\bar{T}_{0}\left(L_{0}\right)=\frac{1}{k_{0}} \bar{G}_{0}\left(L_{0}, L_{0}\right) \bar{q}_{10}+\bar{B}_{0}\left(L_{0}\right)
$$

In layer $i ; i=1,2, \ldots, N$ : the interface temperatures are

$$
\begin{gathered}
\bar{T}_{i}(0)=\frac{1}{k_{i}} \bar{G}_{i}(0,0) \bar{q}_{i-1, i}+\frac{1}{k_{i}} \bar{G}_{i}\left(0, L_{i}\right) \bar{q}_{i+1, i} \\
\bar{T}_{i}\left(L_{i}\right)=\frac{1}{k_{i}} \bar{G}_{i}\left(L_{i}, 0\right) \bar{q}_{i-1, i}+\frac{1}{k_{i}} \bar{G}_{i}\left(L_{i}, L_{i}\right) \bar{q}_{i+1, i}
\end{gathered}
$$

In the last layer $(N)$, the temperatures at the interfaces are

$$
\bar{T}_{N}(0)=\frac{1}{k_{N}} \bar{G}_{N}(0,0) \bar{q}_{N-1, N}
$$

$$
\bar{T}_{N}\left(L_{N}\right)=\frac{1}{k_{N}} \bar{G}_{N}\left(L_{N}, 0\right) \bar{q}_{N-1, N}
$$

Here, the Green's function $\bar{G}_{i}$ for the Fourier-space response of a layer with slug flow is available in the form of complex-valued exponentials [19] and is given in the Appendix.

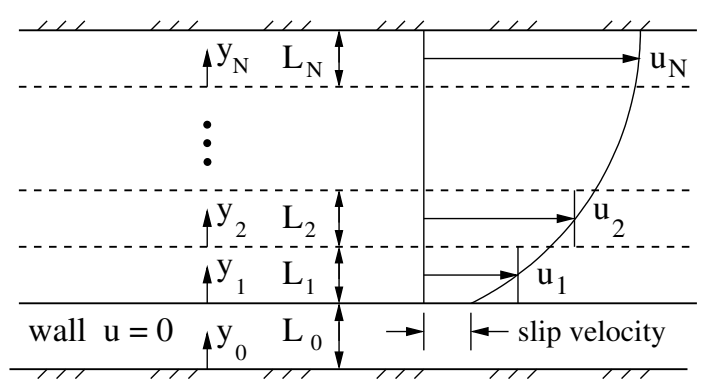

Fig. 3 Multilayer model with spatially uniform fluid velocity in each of $N$ fluid layers and with zero velocity in the stationary wall (layer 0 ). 
In the preceding temperature expressions, all of the interface heat fluxes are initially unknown. The heat flux leaving one layer enters the adjacent layer, $\bar{q}_{i-1, i}=-\bar{q}_{i, i-1}$ and the temperature in adjacent layers is equal at each interface internal to the fluid

$$
\bar{T}_{i}(0)=\bar{T}_{i-1}\left(L_{i-1}\right) ; \quad i=2,3 \ldots, N
$$

The temperature jump condition associated with slip flow at the fluid-solid interface may be written as

$$
\bar{T}_{1}(0)-\bar{T}_{0}\left(L_{0}\right)=R_{0} \bar{q}_{10}
$$

where $R_{0}=r K n L / k_{1}$ is the contact resistance $\left(\mathrm{m}^{2} \mathrm{~K} / \mathrm{W}\right)$. Next, Eqs. (15-19) are combined with Eq. (20) to eliminate temperature. The result is a set of $N$ linear algebraic equations for the unknown heat fluxes between the layers, which may be stated in matrix form

$$
\begin{aligned}
& {\left[\begin{array}{ccccc}
C_{0}+C_{1}+R_{0} & -D_{1} & 0 & \ldots & 0 \\
-D_{1} & C_{1}+C_{2} & -D_{2} & \ldots & 0 \\
0 & -D_{2} & C_{2}+C_{3} & & \vdots \\
\vdots & \vdots & & \ddots & -D_{N-1} \\
0 & 0 & \ldots & -D_{N-1} & C_{N-1}+C_{N}
\end{array}\right]} \\
& \times\left[\begin{array}{c}
\bar{q}_{10} \\
\bar{q}_{21} \\
\bar{q}_{32} \\
\vdots \\
\bar{q}_{N, N-1}
\end{array}\right]=\left[\begin{array}{c}
-\bar{B}_{0}\left(L_{0}\right) \\
0 \\
0 \\
\vdots \\
0
\end{array}\right]
\end{aligned}
$$

Symbols $C_{i}$ and $D_{i}$ used in the preceding expression are given next

$$
\begin{aligned}
C_{i} & =\frac{1}{k_{i}} \bar{G}_{i}(0,0)=\frac{1}{k_{i}} \bar{G}_{i}\left(L_{i}, L_{i}\right) \\
D_{i} & =\frac{1}{k_{i}} \bar{G}_{i}\left(0, L_{i}\right)=\frac{1}{k_{i}} \bar{G}_{i}\left(L_{i}, 0\right)
\end{aligned}
$$

For any multilayered system, it is now possible to calculate the $N$ unknown heat fluxes $\bar{q}_{i j}$ through all interfaces in the system. Cramer's rule may be used to solve for $q$ for a slip flow and adjacent wall approximated by two or three layers. However, for four or more layers, the well-known tridiagonal algorithm may be used. Once the heat fluxes are found, the temperature at any interface is given by Eq. (15-19).

In the present work, the heat transfer is caused by heating in the wall and is manifest in the preceding matrix equation as term $\bar{B}_{0}\left(L_{0}\right)$ on the right-hand side. The specific form of heating function $\bar{B}_{0}\left(L_{0}\right)$ is given in the Appendix. Viscous dissipation, not present in the preceding formulation, could be easily added by including a heating term for viscous dissipation for each fluid layer into the right-hand side of the preceding matrix equation.

\section{Spatial Average Temperature}

The preceding temperature expression for the temperature at a point is valuable for understanding the thermal mechanisms present in microchannel flow. However, the average temperature over a finite region on the interface is important in microchannels to represent a finite-sized temperature sensor, either at the heating location or in a region immediately downstream. Such a heated region and its downstream neighbor region could represent two neighboring electronic circuit elements, one introducing heat and one subject to that heat.

Of interest is the spatial average temperature on a region $(\xi<x<\xi+a)$ of the fluid-solid interface. This average temperature may be computed from the wall temperature by

$$
\begin{aligned}
T_{\mathrm{av}}(\xi, \omega) & =\frac{1}{a} \int_{\xi}^{\xi+a} T_{0}\left(x, L_{0}, \omega\right) \mathrm{d} x \\
& =\frac{1}{a} \int_{\xi}^{\xi+a}\left[\frac{1}{2 \pi} \int_{-\infty}^{\infty} \bar{T}_{0}\left(\beta, L_{0}, \omega\right) e^{j \beta x} \mathrm{~d} \beta\right] \mathrm{d} x \\
& =\frac{1}{2 \pi} \int_{-\infty}^{\infty} \bar{T}_{1}\left(\beta, L_{0}, \omega\right)\left[\frac{e^{j \beta \xi}\left(e^{j \beta a}-1\right)}{j \beta a}\right] \mathrm{d} \beta
\end{aligned}
$$

Here, the spatial integral over $x$ has been carried out in closed form. Quantity $T_{\mathrm{av}}$ is actually easier to evaluate numerically, compared to $T_{1}$, because of the introduction of factor $1 / \beta$, which causes the integrand to vanish more rapidly as $\beta \rightarrow \pm \infty$. Average temperatures computed from the preceding expression will be reported later in this paper for the heater $(\xi=0)$ and for a region located downstream of the heater $(\xi>a)$.

\section{Nusselt Number}

In this section, the development of the Nusselt number is given. The Nusselt number is the dimensionless heat transfer coefficient, defined by

$$
N u(x)=h(x) L / k_{1} ; \quad h(x)=\frac{q_{0}}{T_{w}(x)-T_{m}(x)}
$$

Here, $q_{0}$ is the heater-supplied heat flux, $T_{w}$ is the local temperature on the heater, and $T_{m}$ is the fluid mean temperature. It is important to note that the supplied heat flux $q_{0}$ is used to define $h$ because it is experimentally measurable. Traditional definitions of $h$ involve the local flux into the fluid, which is difficult to observe at the microscale, particularly when wall conduction is present.

The fluid mean temperature is found from a velocity-weighted average temperature in the fluid. For the parallel-plate channel, the mean temperature is defined in Fourier space by

$$
\bar{T}_{m}(\beta)=\frac{2}{U L} \int_{y=0}^{L / 2} u(y) \bar{T}(\beta, y) \mathrm{d} y
$$

where $u(y)$ is the local velocity, $U$ is the average velocity in the channel, and the half-channel height is $L / 2$. For the layered description of the fluid used here, the single integral across the channel may be replaced by a series of integrals over each layer

$$
\bar{T}_{m}(\beta)=\frac{2}{U L} \sum_{i=1}^{N} u_{i} \int_{y_{i}=0}^{L_{i}} \bar{T}_{i}\left(\beta, y_{i}\right) \mathrm{d} y_{i}
$$

where $\bar{T}_{i}$ is the temperature and $u_{i}$ is the uniform velocity in layer $i$. If the layers are sufficiently small, then the integral across each layer may be replaced, to good approximation, with the simple average of the temperatures at the two boundaries of each layer. That is

$$
\bar{T}_{m}(\beta)=\frac{2}{U L} \sum_{i=1}^{N} u_{i} L_{i} \frac{1}{2}\left(\bar{T}_{i}(\beta, 0)+\bar{T}_{i}\left(\beta, L_{i}\right)\right)
$$

These layer-boundary temperatures are important because they may be computed at little cost from already known quantities $\bar{q}_{m n}, C_{i}$, and $D_{i}$.

The local Nusselt number must be computed from the real-space temperature difference $\left[T_{w}(x)-T_{m}(x)\right]$, and the average heat transfer coefficient (or equivalently, the average Nusselt number) must be computed from the following integral

$$
N u_{\text {av }}=\frac{1}{a} \int_{0}^{a} \frac{h(x) L}{k_{1}} \mathrm{~d} x=\frac{L}{a k_{1}} \int_{0}^{a} \frac{q_{0}}{T_{w}(x)-T_{m}(x)} \mathrm{d} x
$$

It is important to note that because the Fourier transform is a linear operator applied to temperature, the Nusselt number cannot be computed in transform space and then inverse transformed. Numerical values for the local and average Nusselt number are reported later. 


\section{Numerical Considerations}

Some care was needed to obtain efficient evaluation of the Fourierinversion integral in Eq. (9), which is an improper integral (limits at infinity). The improper integral on $0<\beta<\infty$ was replaced by a summation of proper integrals, each of width $\pi$, beginning at $\beta=0$. Additional terms of this series were added until the fractional change in the magnitude of the running sum was less than a tolerance to provide five-digit precision. The integral over $-\infty<\beta<0$ was handled in a similar way. All coding was carried out with variables of type double-precision complex in FORTRAN 95.

The precision associated with number of layers in the fluid was investigated by computing the average Nusselt on the heater for a single specific geometry with $10,20,40$, and 60 layers in the fluid. The specific geometry is $K n=0, a / L=20, w / L=200$ for airflow over a glass wall (refer to Table 1). The percentage change in the wall temperature shows that 10 fluid layers give precision within $0.74 \%$, compared to the 60-layer calculation at a flow value of $P e=100$ and within $0.70 \%$ at $P e=1$. Based on this information, 10 fluid layers were used for all the numerical results presented in this paper. Nonuniform spacing of the fluid layers was used, with thicker layers farther from the wall, according to $L_{i} / L \approx i^{1.5}$, in order to equalize the velocity jump across successive layers. The precision associated with the discrete approximation to the velocity distribution was computed at the worst case laminar flow condition of $K n=0$. The precision will improve for $K n>0$ because there is less velocity variation across the channel when slip flow is present.

\section{Results}

The normalized temperature discussed in this section is normalized amplitude of temperature, in the form $T^{+}=\left[T(x, y, \omega)-T_{0}\right]$. $k_{1} /\left(q_{0} a\right)$, where $T_{0}$ is the ambient temperature, $k_{1}$ is the fluid conductivity, $q_{0}$ is the introduced heat flux, and $a$ is the axial length of the heated region. In the following sections, the temperature in the microchannel is explored for various gas-flow rates, varying slipflow effects, and different wall materials. The base case chosen for analysis is in a microchannel with a thin heater size $a / L=20$ located on the interface between the airflow and a glass (or ceramic) wall of thickness $w / L=200$. This geometry could represent, as one example, a flow channel $2.5 \mu \mathrm{m}$ in height with a heater of streamwise extent $50 \mu \mathrm{m}$ on a wall of $500-\mu \mathrm{m}$ thickness. Thermal properties of the gas and wall materials are given in Table 2 . The temperature-jump parameter, Eq. (8), is $r=1.667$, which is a typical value for air [3] ]. The heating frequency is $\omega^{+}=1.0$.

Table 1 Percent change in average Nusselt number on the heater caused by lowering the number of fluid layers ${ }^{\mathrm{a}}$

\begin{tabular}{crrrc}
\hline \hline Peclet & \multirow{2}{*}{$N u(60$ layer $)$} & \multicolumn{3}{c}{$\%$ change } \\
\cline { 3 - 5 } & & 40 layer & 20 layer & 10 layer \\
\hline 1 & 2687.75 & 0.0249 & 0.1596 & 0.6987 \\
10 & 2499.65 & 0.0252 & 0.1608 & 0.7037 \\
100 & 689.63 & 0.0264 & 0.1685 & 0.7371 \\
\hline \hline
\end{tabular}

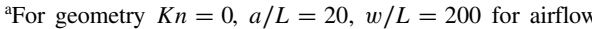
over glass at three Peclet values. The basis for comparison is the Nusselt number for the 60-layer case, also listed.

Table 2 Thermal property ratios used in simulations ${ }^{\mathrm{a}}$

\begin{tabular}{lrcrrr}
\hline \hline Material & $k_{0}\left(\frac{\mathrm{W}}{\mathrm{mK}}\right)$ & $\alpha_{0}\left(\frac{\mathrm{m}^{2}}{\mathrm{~s}}\right)$ & \multicolumn{1}{c}{$\frac{k_{0}}{k_{1}}$} & $\frac{\alpha_{0}}{\alpha_{1}}$ & $\frac{(\rho c)_{0}}{(\rho c)_{1}}$ \\
\hline Plastic & 0.23 & $0.10\left(10^{-6}\right)$ & 8.75 & 0.00444 & 1984 \\
Glass & 1.38 & $0.83\left(10^{-6}\right)$ & 52.47 & 0.03688 & 1420 \\
Stainless steel & 13.00 & $3.40\left(10^{-6}\right)$ & 494.30 & 0.15111 & 3279 \\
\hline \hline
\end{tabular}

avalues for air are $k_{1}=0.0263 \mathrm{~W} / \mathrm{m} / \mathrm{K}, \alpha_{1}=22.5\left(10^{-6}\right) \mathrm{m}^{2} / \mathrm{s}$, and $(\rho c)=1169 \mathrm{~J} / \mathrm{m}^{3} / \mathrm{K}$
Table 3 Spatial average heat transfer coefficient (normalized) on the heated region for airflow over three wall materials at several airflow (Peclet) values, without slip flow $(K n=0)$ and with slip flow $(K n=0.04)$

\begin{tabular}{lrrc}
\hline \hline Wall & $P e$ & $K n=0$ & $K n=0.04$ \\
\hline Plastic & 20 & 1111.7 & 885.7 \\
& 40 & 849.4 & 679.1 \\
& 60 & 576.6 & 469.9 \\
& 80 & 416.0 & 353.1 \\
& 200 & 217.6 & 202.6 \\
Glass & 500 & 169.4 & 162.8 \\
& 20 & 2282.0 & 1819.8 \\
& 40 & 1734.5 & 1388.9 \\
& 60 & 1180.5 & 965.9 \\
& 80 & 859.9 & 731.1 \\
& 200 & 450.8 & 419.7 \\
Steel & 500 & 349.5 & 336.2 \\
& 20 & 10443.4 & 8344.7 \\
& 40 & 7856.4 & 6316.2 \\
& 60 & 5361.2 & 4415.9 \\
& 80 & 3963.8 & 3391.9 \\
& 200 & 2107.0 & 1960.3 \\
& 500 & 1623.8 & 1526.7 \\
\hline \hline
\end{tabular}

\section{A. Spatial Average Temperature on Heater}

In this section, the average temperature on the heater is discussed, starting with the base case of airflow over a glass wall of thickness $w / L=200$, heater size $a / L=20$, for heating at frequency $\omega^{+}=1$. The temperature on the heater for this case is plotted vs Peclet number (fluid flow rate) in Fig. 4.

Figure $\underline{4}$ shows the effect of different wall materials on the heater temperature, specifically walls made of plastic, glass, and steel. Plastic, with the lowest thermal conductivity, has the highest temperature, and steel with the highest conductivity, has the lowest temperature (normalized by the introduced heat flux). The temperature values are almost completely flat, that is, little affected by convection, except for the plastic wall case, which decreases slightly at the higher Peclet values. The effect of slip flow on temperature is so slight as to be nearly invisible in Fig. 4, and then only for plastic at higher Peclet values. The temperature for the plastic wall case changes by $0.26 \%$ from $K n=0$ to 0.04 at $P e=500$. Although the heater temperature is insensitive to fluid flow, in the next section, the heat transfer coefficient is shown to be strongly flow dependent.

\section{B. Nusselt Number on Heater}

In this section, results are presented for the Nusselt number. It is important to note that the Nusselt number trend in microchannel flow with wall conduction is very different from macroscale flow. In microchannel flow, when the Peclet number is small, the heat conducted through the channel wall (which moves in all directions) dominates over the heat conducted by the fluid (which is in the direction of fluid velocity). The wall conduction prewarms the fluid upstream of the heater, which decreases the temperature difference between the fluid and the wall [19]. As the Peclet number increases, convection has more influence on the heat transfer so that the temperature difference between the fluid and the wall increases, which decreases the Nusselt number. Another view of this behavior is that the wall temperature shown in Fig. 4 is nearly constant as the Peclet number changes, therefore all of the variation in Nusselt number comes from changes in the fluid temperature.

Figure 5 shows the spatial average Nusselt number on the heater as a function of Peclet number for airflow over three different wall materials and the effect of slip flow is also included. Corresponding numerical values of the average Nusselt number are given in Table 3 . The curves in Fig. 5 all have the same shape, starting at low Peclet values with a small slope and with negative curvature (Nusselt number decreases as Peclet increases). At slightly higher Peclet values, the Nusselt values begin to decrease more rapidly and then the 


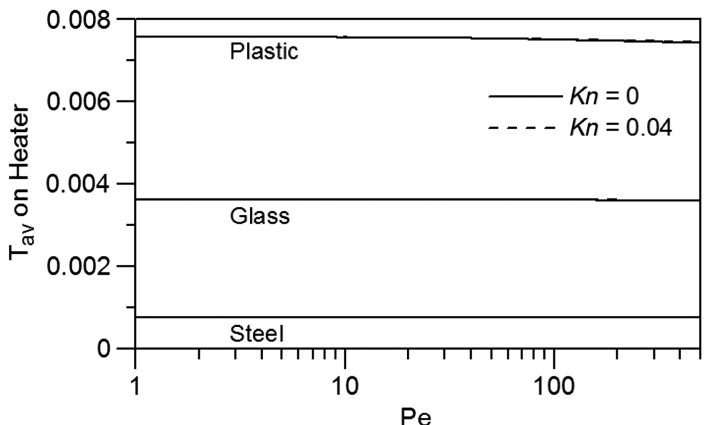

Fig. 4 Average temperature on the heater (normalized) vs $P e$ for airflow over plastic, glass, and steel walls.

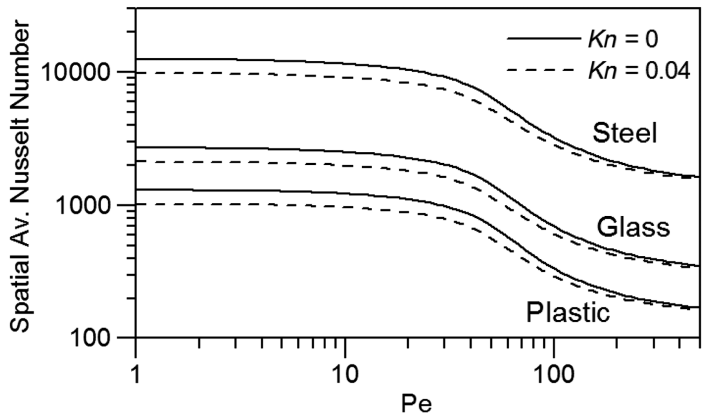

Fig. 5 Average Nusselt number on the heater for airflow over plastic, glass, and steel walls.

curvature passes through an inflection point around $P e=70$ and the rate of decrease slows.

The effect of wall material is in order of wall conductivity, with high-conductivity steel having the highest Nusselt numbers, followed by the glass and plastic wall. The effect of slip flow, shown in Fig. 5, is that the Nusselt number on the heater is always lower when slip flow is present. This is caused by the slip-flow temperature jump at the wall producing a larger wall-fluid temperature difference. Because the Nusselt number is proportional to the inverse of this temperature difference, slip flow pushes the Nusselt number lower.

To further explore the interplay between convection and wall conduction, Fig. 6 shows the spatial variation of the Nusselt number along the heater $(0<x / a<1)$ for several values of the Peclet number, for airflow with and without the presence of slip flow, for the base case of airflow over a glass wall. First, the effect of slip flow has the same trend for all the curves shown in Fig. 5, which is to lower the Nusselt number relative to continuum $(K n=\overline{0})$ flow. Next, the shape of the curves in Fig. 6 will be examined. For all of the curves shown in Fig. $\underline{6}$, there is a small region near $x=0$ where $N u(x)$ falls from about value 700 to a local minimum. This region is followed by an increasing Nusselt region with uniform slope. The values of the uniform slope vary inversely with Peclet number, with the larger slope occurring for the smallest Peclet value. For the $P e=10,20$, and 40 curves, the uniform slope region is followed by a knee where

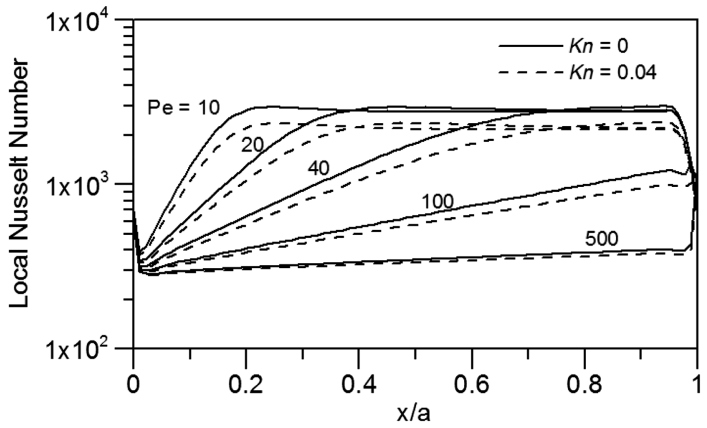

Fig. 6 Local Nusselt number version position on the heater for airflow over a glass wall for several Peclet values. the rising trend is arrested and a plateau in Nusselt value is achieved; note that the plateau Nusselt value is the about same for these three Peclet values. For $P e=10$, the knee appears at about $x / a=0.15$, for $P e=40$ the knee has moved downstream to $x / a=0.35$, and for $P e=40$ the knee has moved to $x / a=0.7$. In contrast, at $P e=100$, the knee is missing and the Nusselt value does not reach the plateau value. For the largest $P e$ value shown in Fig. $6(P e=500)$, the uniform slope region extends across the heater, the $\bar{N}$ usselt value does not reach the plateau value, and the knee is replaced by an upward jump in the Nusselt value just at the end of the heater. Clearly, the shape of the Nusselt curve shows large changes over this range of Peclet number. Conduction in the wall provides an explanation for the different trends at the downstream end of the heater. Briefly, there is a transition in the temperature downstream of the heater that occurs around $P e=70$, and wall conduction causes this downstream event to be visible on the downstream end of the heater. In the next section, the temperature downstream of the heater is given to provide a window into this behavior.

\section{Region Downstream of Heater}

In this section, results for the temperature are presented at a location downstream of the heater. This region could represent a multicomponent electronic device with an unheated downstream region affected by a heated upstream region. Alternately, this downstream region could represent a temperature sensor in a microfluidic system.

Figure 7 shows the average temperature in the downstream region $1.15<x \overline{/ a}<2.15$ for a range of $P e$ values and for several $K n$ values. Recall that the heater is located in region $0<x / a<1$.0. In Fig. 7, the principal feature of the average temperature in the downstream region, as Peclet rises, is that temperature rises up to some plateau. Recall that for a fixed geometry, $P e$ is proportional to mean gas velocity. The rising trend in temperature with Peclet number is caused by convection carrying an increasing amount of heat from the upstream heater to this downstream observation location. In gas flows for this range of $P e$ values, axial conduction in the wall is dominant over convection. The extreme example is the steel wall curve that starts out flat because it takes a great deal of gas flow to affect the steel wall temperature. The plateau in temperature at higher $P e$ occurs when the convection begins to overcome the wall conduction.

The effect of $K n$ on the downstream location, shown in Fig. 7, is caused by the temperature jump at the wall that is present in slip flow. As $K n$ rises, the wall temperature jump produces a different effect for small $P e$ and large $P e$. At small $P e$ values, as $K n$ rises, the average wall temperature rises, because heat is moving from the wall to the fluid across the slip-flow temperature jump. The wall is hotter than the fluid because of wall conduction, again for small $P e$ values. This is true for both the plastic and glass wall cases in Fig. 7; however, for the steel wall, the effect is too small to be seen. At larger $P e$ values $(P e>70)$, the effect of rising $K n$ has the opposite effect on the wall temperature. Here, the wall temperature falls as $K n$ rises because at this downstream location, heat is flowing from the fluid to the wall across the slip-flow temperature jump. The fluid is hotter than the wall because of convection from the upstream heater.

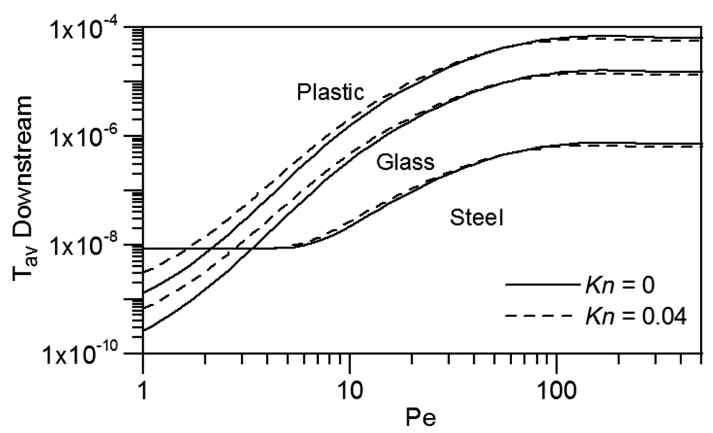

Fig. 7 Average temperature over an unheated downstream region $1.15<x / a<2.15$ for airflow over plastic, glass, and steel walls. 
It is important to note that the shape of the temperature curves in Fig. 7 are unique to wall-dominated heat transfer present in microchannel flow. This shape is a consequence of a transition in the shape of the spatial temperature distribution (not shown) in the neardownstream region as Peclet rises. At low Peclet values, the spatial temperature distribution is defined by wall conduction and is symmetric about the heater. As Peclet rises, the temperature transitions to a convection-dominated shape with a downstream-biased thermal footprint. This point is discussed more fully in a previous paper by the authors on liquid flow in microchannels [19].

\section{Conclusions}

In this paper, the analytical solution for the heat transfer in a parallel-plate microchannel is given in the slip-flow regime with thermally participating walls. Results are given for a wide range of $P e$, at several $K n$ values, for three wall materials in airflow. The heater temperature is dominated by wall conduction for all but the largest airflow values studied. The effect of slip flow is to raise the heater temperature, caused primarily by the temperature jump at the wall that is proportional to the $K n$ value. As the heat transfer coefficient is proportional to the inverse of temperature, the addition of slip flow reduces the average heat transfer coefficient on the heater. The spatial distribution of the heat transfer coefficient changes from a curved-downward shape to a curved-upward shape as the Peclet number rises.

Downstream of the heater at low gas flows, the wall temperature is larger than the fluid temperature similar to the trend on the heater, and the range of Peclet values defining this behavior depends on the interplay of axial wall conduction and fluid convection. The effect of slip flow in the downstream region is to raise the wall temperature at low gas flows, similar to that of the heater itself, because heat is flowing from the wall into the fluid across the slip-flow temperature jump. At higher gas flows, however, the near-downstream region experiences a transition to where the wall temperature falls below the fluid temperature and heat carried by the flow is entering the wall. After this transition, which occurs around $P e=70$, the effect of slip flow is to lower the wall temperature (relative to laminar flow), again because heat flows across a temperature jump at the wall-fluid interface. Application of these results includes thermal effects and thermal processing in microfluidic systems.

\section{Appendix: Distribution of Heat in the Wall}

In this Appendix, the form of the volume heating term is discussed. This term comes from applying the Fourier transform, Eq. (9), to the real-space heating integral, Eq. (6), to find

$$
\bar{B}_{0}\left(y_{0}\right)=\frac{1}{k_{0}} \int_{y^{\prime}=0}^{L_{0}} \bar{g}\left(\beta, y_{0}^{\prime}\right) \bar{G}_{0}\left(\beta, y_{0}, y_{0}^{\prime}\right) \mathrm{d} y_{0}^{\prime}
$$

Here, $\bar{g}$ is the Fourier-space heating distribution in the wall and $\bar{G}_{0}$ is the Fourier-space Green's function for layer 0. The Fourier-space Green's function for a uniform velocity layer with homogeneous type 2 boundaries is given by [20]

$$
\bar{G}\left(y, y^{\prime}\right)=\frac{\left(e^{-\nu\left(2 L-\left|y-y^{\prime}\right|\right)}+e^{-\nu\left(2 L-y-y^{\prime}\right)}\right)}{2 \nu\left(1-e^{-2 \nu L}\right)}+\frac{\left(e^{-\nu\left(\left|y-y^{\prime}\right|\right)}+e^{-\nu\left(y+y^{\prime}\right)}\right)}{2 \nu\left(1-e^{-2 \nu L}\right)}
$$

Note that the subscript identifying the layer number has been suppressed. Here, $\nu^{2}=\beta^{2}+j \beta u / \alpha$ and $u$ is the velocity of the layer; in the wall (layer 0 ), the velocity is simply zero. Next, the volume heating function will be given for two heating geometries in the wall, a thin heater at the fluid-wall interface, and heating distributed through the wall thickness.

\section{A1 Thin Heater at Fluid-Wall Interface}

If the heating is confined to the surface of the wall at the fluid-wall interface, then the heat distribution along a short axial region of the wall has the form

$$
g\left(x, y_{0}^{\prime}\right)=\delta\left(y_{0}-L_{0}\right) \times \begin{cases}q_{0} & \text { if } 0<x<a \\ 0 & \text { otherwise }\end{cases}
$$

Here, $q_{0}$ is the introduced heat flux $\left(\mathrm{W} / \mathrm{m}^{2}\right)$. Note that the Dirac delta function is nonzero only at boundary $y_{0}=L_{0}$. Now apply the Fourier transform, Eq. (9), to this heating function to obtain

$$
\bar{g}\left(\beta, y_{0}^{\prime}\right)=\delta\left(y_{0}-L_{0}\right) \frac{q_{0}}{j \beta}\left(1-e^{-j \beta a}\right)
$$

Now combine the preceding expression for the heating function with the Green's function, Eq. (르), and the heating integral, Eq. (1ㅡ), to obtain

$$
\bar{B}_{0}\left(L_{0}\right)=\frac{1}{k_{0}} \frac{q_{0}}{j \beta \nu}\left(1-e^{-j \beta a}\right) \times \frac{1+e^{-2 \nu L_{0}}}{1-e^{-2 \nu L_{0}}}
$$

Note that the Dirac delta function has been used to eliminate the integral on $y^{\prime}{ }_{0}$, and that this expression has been evaluated at location $y_{0}=L_{0}$ as needed in the matrix solution, Eq. (22). The preceding heating function for the thin heater has been studied previously for laminar flow [19].

\section{A2 Heating Distributed Through Wall Thickness}

If the heating is uniform through the wall thickness, then the heat distribution along a short axial region of the wall has the form

$$
g\left(x, y^{\prime}\right)= \begin{cases}q_{0} / L_{0} & \text { if } 0<x<a \\ 0 & \text { otherwise }\end{cases}
$$

Here, $q_{0}$ is the rate of heat introduced per unit area of wall so that $q_{0} / L_{0}$ is the rate of heat per unit volume. The Fourier transform of this distribution is given by

$$
\bar{g}\left(\beta, y_{0}^{\prime}\right)=\frac{q_{0}}{j \beta L_{0}}\left(1-e^{-j \beta a}\right)
$$

Replacing the preceding expression for the heating function and the Green's function, Eq. (A2), into the heating integral, Eq. (A1), and upon evaluation of the integral, the heating function is given by

$$
\bar{B}_{0}\left(L_{0}\right)=\frac{1}{k_{0}} \frac{q_{0}}{j \beta L_{0}}\left(1-e^{-j \beta a}\right) \frac{1}{\nu^{2}}
$$

Note that the spatial integral of the Green's function reduces to the term $1 / \nu^{2}[\underline{21}]$.

\section{Acknowledgment}

The authors would like to acknowledge support from the University of Nebraska under the Undergraduate Creative Activities and Research Experiences program, and from the National Science Foundation through grant CBET 1250625 under program officer Sumanta Acharya.

\section{References}

[1] Jeong, H. E., and Jeong, J. T., "Extended Graetz Problem Including Streamwise Conduction and Viscous Dissipation in Microchannels," International Journal of Heat and Mass Transfer, Vol. 49, No. 13, 2006, pp. 2151-2157.

[2] Cetin, B., Yazicioglu, A. G., and Kakac, S., "Fluid Flow in Microtubes with Axial Conduction Including Rarefaction and Viscous Dissipation," International Communications in Heat and Mass Transfer, Vol. 35, No. 5, 2008, pp. 535-544. 
[3] Cetin, B., Yazicioglu, A. G., and Kakac, S., "Slip-Flow Heat Transfer in Microtubes with Axial Conduction and Viscous Dissipation-An Extended Graetz Problem," International Journal of Thermal Sciences, Vol. 48, No. 9, 2009, pp. 1673-1678.

[4] Davis, E. J., and Gill, N. W., "The Effects of Axial Conduction in the Wall on Heat Transfer with Laminar Flow," International Journal of Heat and Mass Transfer, Vol. 13, No. 3, 1970, pp. 459-470.

[5] Mori, S., Kawamura, Y., and Tanimoto, A., "Conjugated Heat Transfer to Laminar Flow with Internal Heat Source in a Parallel Plate Channel," The Canadian Journal of Chemical Engineering, Vol. 57, No. 6, 1979, pp. 698-703.

[6] Maranzana, G., Perry, I., and Maillet, D., "Mini- and Micro-Channels: Influence of Axial Conduction in the Walls," International Journal of Heat and Mass Transfer, Vol. 47, No. 17, 2004, pp. 3993-4004.

[7] Kroeker, C. J., Soliman, H. M., and Ormiston, S. J., "Three-Dimensional Thermal Analysis of Heat Sinks with Circular Cooling MicroChannels," International Journal of Heat and Mass Transfer, Vol. 47, No. 22, 2004, pp. 4733-4744.

[8] Li, J., Peterson, G. P., and Cheng, P., "Three-Dimensional Analysis of Heat Transfer in a Micro-Heat Sink with Single Phase Flow," International Journal of Heat and Mass Transfer, Vol. 47, No. 19, 2004, pp. 4215-4231.

[9] Kim, S. J., and Kim, D. K., "Forced Convection in Microstructures for Electronic Equipment Cooling," Journal of Heat Transfer, Vol. 121, No. 3, 1999, pp. 639-645.

[10] Kim, D. K., and Kim, S. J., "Averaging Approach for Microchannel Heat Sinks Subject to the Uniform Wall Temperature Condition," International Journal of Heat and Mass Transfer, Vol. 49, No. 3, 2006, pp. $695-706$

[11] Nonino, C., Savino, S., Giudice, S. D., and Mansutti, L., "Conjugate Forced Convection and Heat Conduction in Circular Microchannels,"
International Journal of Heat and Fluid Flow, Vol. 30, No. 5, 2009, pp. 823-830.

[12] Kosar, A., "Effect of Substrate Thickness and Material on Heat Transfer in Microchannel Heat Sinks," International Journal of Thermal Sciences, Vol. 49, No. 4, 2010, pp. 635-642.

[13] Cetin, B., Travis, B. E., and Li, D., "Analysis of the Electro-Viscous Effects on Pressure-Driven Liquid Flow in a Two-Section Heterogeneous Microchannel," Electrochimica Acta, Vol. 54, No. 2, 2008, pp. 660-664.

[14] Morini, G. L., "Scaling Effects for Liquid Flows in Microchannels," Heat Transfer Engineering, Vol. 27, No. 4, 2006, pp. 64-73.

[15] Colin, S., "Gas Microflows in the Slip Flow Regime: A Critical Review on Convection Heat Transfer," Journal of Heat Transfer, Vol. 134, No. 2, 2012, Paper 020908.

[16] Cole, K. D., "Steady-Periodic Heating in Parallel-Plate Microchannel Flow with Participating Walls," International Journal of Heat and Mass Transfer, Vol. 53, No. 5, 2010, pp. 870-878.

[17] Inman, R. M., "Heat Transfer in Thermal Entrance Region with Laminar Slip Flow Between Parallel Plates at Unequal Temperatures," NASA, TN-D-2980, 1965.

[18] Cole, K. D., Beck, J. V., Haji-Sheikh, A., and Litkouhi, B., "Heat Conduction Using Green's Functions," 2nd ed., Taylor and Francis, New York, 2011, pp. 72-75.

[19] Cole, K. D., and Cetin, B., "The Effect of Axial Conduction on Heat Transfer in a Liquid Microchannel Flow," International Journal of Heat and Mass Transfer, Vol. 54, No. 11, 2011, pp. 2542-2549.

[20] Cole, K. D., "Green's Function Library," http://www.greensfunction.unl .edu [retrieved $10 \mathrm{Feb}$. 2013].

[21] Crittenden, P. E., and Cole, K. D., "Fast-Converging Steady-State Heat Conduction in the Rectangular Parallelepiped," International Journal of Heat and Mass Transfer, Vol. 45, No. 17, 2002, pp. 3585-3596. 